\title{
Kontroversies, aversies, inversies in versies
}

\section{Kyk die mens}

\section{Picasso kyk}

\section{Mens en bul}

Blinde woesteling! Jy, Dood, komaan!

Storm, deurboor, hier's my bloed, my bestaan!

My Iyf swenk, my slank hand

wuif die doek elegant-

Kyk, onnosel! Die mens! Ek bly staan!

\section{Minotaur}

Watter god het my, bulmens, kolos, uit my donker gevat, bulkend kom los in dié eeu, dié doolhof,

blind in lig, lomp, geskof?

Vroue is kos, hulle kan nie verlos.

\section{Laat ontdekkings}

I letie, letie, waar is jy?

(Mi langt na di, geselle mijn - Egidiuslied)

$\mathrm{Na}$ 'n lange astrale geswerf

draal ' $n$ jogi weer terug na die werf

van die Heerlike Self

in sy ruim kopgewelf,

maar hy vind hy't intussen gesterf.

\section{Ghoeroe troos bejaarde}

$\mathrm{Na}$ ' $n$ lange gedool en betragting:

'Jou geswerf, skades, skandes, veragting?

Jou vermorsing van lewe?

Ek vind: chemies gedrewe

Móét jy kort skiet in elke verwagting,'

\section{Sieh den Mensch! \\ Picasso sieht \\ I Matador}

Blinde Wut! Ja, du, Tod, komm mal an!

Stürme! Durchbohre! Ich hier, fall an!

Mein Leib schwenkt. Schlanke Hand

winkt das Tuch elegant.

Sieh den Mensch, Dümmling! Ich stehe! Der Mann!

\section{Minotaur}

Fremdes Labyrinth. Was mache ich hier,

Ungeheuer, halb Mensch und halb Stier?

Blind taste ich das Licht,

finde mein Dunkel nicht.

Welche Quälgötter taten es mir?

\section{Späte Entdeckungen}

\section{Iti, Iti, wo bist du geblieben?}

(Mi langt na di, geselle mijn - Egidiuslied)

Im astralischen Wandern geborgen,

war ein Jogi gewahr geworden:

"O Ich, Herrlich und Edel!

Es ist Zeit! In den Schädel!"

Dort erfand er sich aber gestorben.

\section{Sachverständiger tröstet Bejahrten}

Mein' Empfindung, nach tiefer Betrachtung?

Deine Schanden, die Schäden, Verachtung?

Sei mal getröstet. $\mathrm{Nu}$,

chemisch getrieben $\mathrm{du}$

Darum täuschest du jede Erwartung.

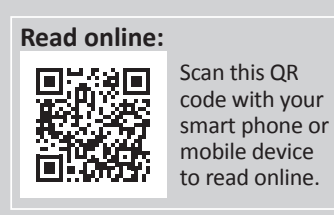

Author: Leendert Dekker

Affiliation: ${ }^{1}$ Private

Corresponding author: Leendert Dekker, leendert@telkomsa.net

How to cite this article: Dekker, L., 2016, Kontroversies, aversies, inversies in versies, Literator 37(1), a1203. http://dx.doi.org/10.4102/lit. v37i1.1203

Note: The Afrikaans limericks are taken from Oos Wes Anapes (ISBN: 978-0-620-59993-1), private publication by Leendert Dekker, 2014. They were freely remade into German by the author himself.

Copyright: (C) 2016. The Authors. Licensee: AOSIS. This work is licensed under the Creative Commons Attribution License. 
Litera

\section{Grafskrif van Hroswitha von Gandersheim:}

ra

ra ra

es

ram

et

in

ram

ii

Oor ons menswees dink ons met $\mathrm{u}$ na,

edel vrou, vroom vorstin: 'Drie maal ra

is jy, ter ra, grond, en gewis

in die grond in $\mathrm{i}$ bis,

sal jy gaan, in grond tot grond vergaan ja.'

\section{Askies vir die wals}

Léon: Voici Ie corps de ballet

La Mort: Voici Ie ballet de corps

- Léon de Querre: Danse macabre

Die ou Hofnar stamp skalks aan Hans Holbeen:

'Nou gaan jý dans, my holste. Kom, hierheen!

Los jou kwassies en messies,

jol met pous en prinsessies!

Ék wals voor. Ék slaat trom. Mét 'n skeenbeen.'

\section{Roswitha von Gandersheim}

Grabschrift für sich selbe

ra

ra

ram

es

et in

ram ram

ii

Deine Frommheit, dein Grab, Roswitha, erzeigt: "Mensch, schau an! Drei Malen ra, ter ra, Erde, du. Gewiss,

in den Boden i bis,

wirst du geh'n, wieder Erde werden ja."

\section{Aufforderung zum Tanze}

Léon: Voici le corps de ballet

La Mort: Voici le ballet de corps

- Léon de Querre: Danse macabre

Hofnarr meldet sich: "Ich bin's, Holbein.

Zeit, O Hohligkeit! Hier, Ringelreihn!

Lass die Pinsel und Bürsten,

waltz mit Nönnchen und Fürsten!

Ích führe, ích trommle, mít 'nem Schienbein." 